\title{
Forced Magnetic Reconnection in Helical Plasmas
}

\author{
Seiya NISHIMURA, Yoshiro NARUSHIMA, Shinichiro TODA, Masatoshi YAGI ${ }^{1,2)}$, Kimitaka ITOH \\ and Sanae -I. ITOH ${ }^{1)}$ \\ National Institute for Fusion Science, Toki 509-5292, Japan \\ ${ }^{1)}$ Research Institute for Applied Mechanics, Kasuga 816-8580, Japan \\ 2) Japan Atomic Energy Agency, Naka 311-0193, Japan
}

(Received 16 September 2010 / Accepted 24 September 2010)

\begin{abstract}
Magnetic islands excited by resonant magnetic perturbations (RMPs) in helical plasmas are investigated. A Rutherford-type equation is coupled with the time evolution equation of the radial electric field associated with the neoclassical particle diffusion due to helically rippled magnetic fields. Using the model, bifurcation between the excitation and the annihilation of non-rotating magnetic islands are newly observed, depending on the magnitude of RMPs and the anomalous plasma viscosity. It is found that the transition between these states is triggered by the change in the radial electric field profile in the vicinity of magnetic island.
\end{abstract}

(C) 2010 The Japan Society of Plasma Science and Nuclear Fusion Research

Keywords: magnetic island, radial electric field, plasma rotation, neoclassical particle diffusion

DOI: $10.1585 /$ pfr.5.040

Forced magnetic reconnection due to resonant magnetic perturbations (RMPs) is of great interest in magnetic fusion plasmas. Bifurcation of equilibria between with and without magnetic islands is observed in helical devices such as the LHD [1,2] and the TJ-II [3], where the tearing mode is linearly stable. In particular, spontaneous annihilation of magnetic islands called 'self-healing' triggers the improvement of plasma confinements. Magnetic islands and stochastic layers in helical plasmas have been investigated in the context of the three-dimensional resistive magnetohydrodynamics (MHD) equilibrium [4], and mechanism of the self-healing is under investigation. Such bifurcation is also observed in tokamaks, and theoretical works reveal that poloidal and toroidal rotations of plasmas play key roles, screening the penetration of RMPs [5]. In helical plasmas, the poloidal rotation is driven by $E \times B$ and diamagnetic drifts, where radial electric fields are produced by the neoclassical particle diffusion associated with helically rippled magnetic fields [6]. Therefore, the poloidal rotation might be associated with the self-healing mechanism. In order to test this hypothesis, theoretical models including finite Lamor radius (FLR) effects, such as the neoclassical particle diffusion, are necessary.

In this paper, we introduce a simple theoretical model describing the forced magnetic reconnection in helical plasmas, and the bifurcation mechanism associated with the poloidal rotation is examined.

The derivation of the model equations is outlined below. We start from two-fluids equations including FLR effects based on the drift ordering [7]. Perpendicular electric current is composed of the polarization current, the diamagnetic current and currents due to anisotropic pres- sure tensors. Neoclassical particle fluxes are perturbatively included by substituting gyrophase-averaged distribution functions given by linearized drift-kinetic equations into moment formulae of anisotropic pressure tensors. We adopt a simplified version of the radial neoclassical particle fluxes given in Ref. [8]. For parallel electric currents, we focus on the regime where the bootstrap current is less important than the inductive current. In fact, the direction of the perturbed bootstrap current is not essential for the bifurcation [1]. Similarly, the direct impact of the polarization current on the magnetic island growth is neglected. RMPs are introduced by finite edge boundary conditions of perturbed magnetic fields. Considering assumptions above, the asymptotic matching of inner and outer boundary layer solutions in the vicinity of the rational surface is performed. The so-called cosine-matching [9] gives a Rutherford-type equation [10], where the island width should be lager than the linear boundary layer width. The so-called sine-matching [11] gives the torque balance, which corresponds to the time evolution equation of the radial electric field. Density and temperature are assumed to be flattened inside magnetic islands at each time step (no diamagnetic drift), due to fast parallel transports. Then, the poloidal rotation of magnetic islands is driven by the $E \times B$ drift at the rational surface (stop in the plasma rest frame).

The model equations are:

$$
\begin{aligned}
& I_{1} \frac{\mathrm{d} w}{\mathrm{~d} t}=-\eta_{\|} c^{2} \Delta_{0}^{\prime}\left(\frac{w_{\mathrm{vac}}^{2}}{w^{2}} \cos \Theta-1\right), \\
& \frac{\partial \Theta}{\partial t}=-\frac{c k_{\theta}}{B_{0}} E_{\mathrm{rs}}
\end{aligned}
$$




$$
\begin{aligned}
\frac{\partial E_{\mathrm{r}}}{\partial t}= & -C_{\mathrm{M}} \Delta_{0}^{\prime} w_{\mathrm{vac}}^{2} w \sin \Theta-\frac{e}{\epsilon_{\perp}}\left(\Gamma_{\mathrm{i}}^{\mathrm{neo}}-\Gamma_{\mathrm{e}}^{\mathrm{neo}}\right) \\
& +\mu_{\mathrm{ano}} \frac{\partial}{\partial r}\left[\frac{1}{r} \frac{\partial}{\partial r}\left(r E_{\mathrm{r}}\right)\right] .
\end{aligned}
$$

where $\{t, r\}$ are the time and the radial position, respectively. $\left\{w, \Theta, E_{\mathrm{r}}\right\}$ are the magnetic island width across the O-point, the poloidal phase angle of the O-point and the radial electric field, respectively. Note that $w$ and $\Theta$ are zero-dimensional, while $E_{\mathrm{r}}$ depends on the radial position. $E_{\mathrm{rs}}$ is given by $E_{\mathrm{rs}}=E_{\mathrm{r}}\left(r_{\mathrm{s}}\right)$, where $r_{\mathrm{s}}$ is the radial position of the rational surface. $I_{1}$ is a numerical constant of order unity [9], $\eta_{\|}$is the parallel resistivity, $c$ is the velocity of light, $w_{\text {vac }}$ is the vacuum island width depending on the magnitude of RMPs, $k_{\theta}$ is the poloidal wave number, $B_{0}$ is the toroidal magnetic field, $e$ is the elementary charge, $\epsilon_{\perp}$ is the electric permittivity and $\mu_{\text {ano }}$ is the anomalous plasma viscosity observed in experiments [12]. $\Delta_{0}^{\prime}<0$ is the tearing mode stability parameter in currentless cylindrical plasmas without RMPs. $C_{\mathrm{M}}$ is associated with the Maxwell stress due to the interaction between magnetic islands and RMPs [13], where $C_{\mathrm{M}}$ is finite (zero) inside (outside) magnetic islands. $\Gamma_{\mathrm{i}}^{\text {neo }}$ and $\Gamma_{\mathrm{e}}^{\text {neo }}$ indicate ion and electron neoclassical particle fluxes give such that

$$
\begin{aligned}
& \Gamma_{\mathrm{i}}^{\text {neo }}=-\frac{C_{1} v_{\mathrm{i}}}{E_{\mathrm{r}}^{2}+C_{2} v_{\mathrm{i}}^{2} r^{2}} p_{\mathrm{i}} T_{\mathrm{i}}\left(\frac{n_{\mathrm{e}}^{\prime}}{n_{\mathrm{e}}}-\frac{e E_{\mathrm{r}}}{T_{\mathrm{i}}}+2 \frac{T_{\mathrm{i}}^{\prime}}{T_{\mathrm{i}}}\right), \\
& \Gamma_{\mathrm{e}}^{\text {neo }}=-\frac{C_{1} v_{\mathrm{e}}}{E_{\mathrm{r}}^{2}+C_{2} v_{\mathrm{e}}^{2} r^{2}} p_{\mathrm{e}} T_{\mathrm{e}}\left(\frac{n_{\mathrm{e}}^{\prime}}{n_{\mathrm{e}}}+\frac{e E_{\mathrm{r}}}{T_{\mathrm{e}}}+2 \frac{T_{\mathrm{e}}^{\prime}}{T_{\mathrm{e}}}\right),
\end{aligned}
$$

where the electrostatic quasi-neutrality is assumed. $n_{\mathrm{e}}$ is the electron density, $\left\{p_{\mathrm{e}}, T_{\mathrm{e}}\right\}$ are the pressure and the temperature of the electron and $\left\{p_{\mathrm{i}}, T_{\mathrm{i}}\right\}$ are those of the ion, respectively. The prime indicates the radial derivative. $\left\{v_{\mathrm{i}}, v_{\mathrm{e}}\right\}$ are the ion and electron collision frequencies, respectively. Coefficients are given by $C_{1}=$ $(15 \sqrt{\pi} / 8)\left(\epsilon_{\mathrm{t}} \epsilon_{\mathrm{h}}^{1 / 2} / 1.67 e^{2}\right)$ and $C_{2}=3 B_{0}^{2} / 1.67 c^{2} \epsilon_{\mathrm{t}} \epsilon_{\mathrm{h}}$, where $\left\{\epsilon_{\mathrm{t}}, \epsilon_{\mathrm{h}}\right\}$ are the inverse aspect ratio of torus and the helical magnetic field modulation rate, respectively.

We numerically solve Eqs. (1)-(5) in the typical parameter of the LHD. Magnetic islands with $(m, n)=(1,1)$ are considered, where $\{m, n\}$ are poloidal and toroidal mode numbers, respectively. Unperturbed equilibrium profiles are given by $q=2.6-1.6\left(r / r_{\mathrm{s}}\right)^{1.3}$ with $r_{\mathrm{s}}=0.85$, $T_{\mathrm{i}}=T_{\mathrm{e}}=2\left(1-(r / a)^{2}\right)+0.2[\mathrm{keV}]$ and $n_{\mathrm{e}}=2 \times 10^{19}(1-$ $\left.(r / a)^{8}\right)+0.2 \times 10^{19}\left[\mathrm{~m}^{-3}\right]$, where $q$ is the safety factor and $a$ is the minor radius. Several models for the flattening of density and temperature profiles inside magnetic islands are examined, however the results are not essentially changed. The radial electric field is negative, i.e. the ion root [12], in the absence of magnetic islands.

The parameter dependence of the saturated island width is examined (Fig. 1). It is found that the saturation state shows the bifurcation, where the unperturbed current sheet and the non-rotating magnetic island are replaced. In a strict sense, the annihilation of magnetic islands indicates that the island width is smaller than the linear boundary
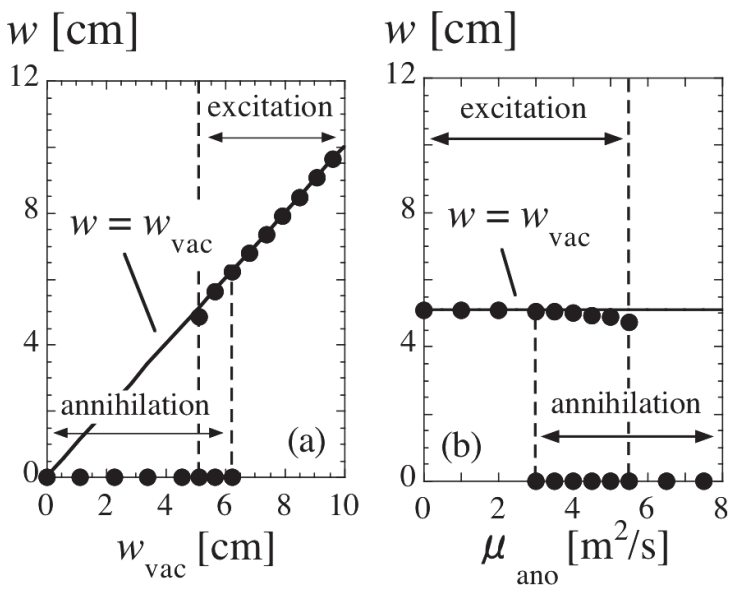

Fig. 1 Dependences of the saturated island width on (a) the vacuum island width $\left(\mu_{\text {ano }}=5 \mathrm{~m}^{2} / \mathrm{s}\right)$ and (b) the anomalous plasma viscosity $\left(w_{\text {vac }}=5.1 \mathrm{~cm}\right)$.

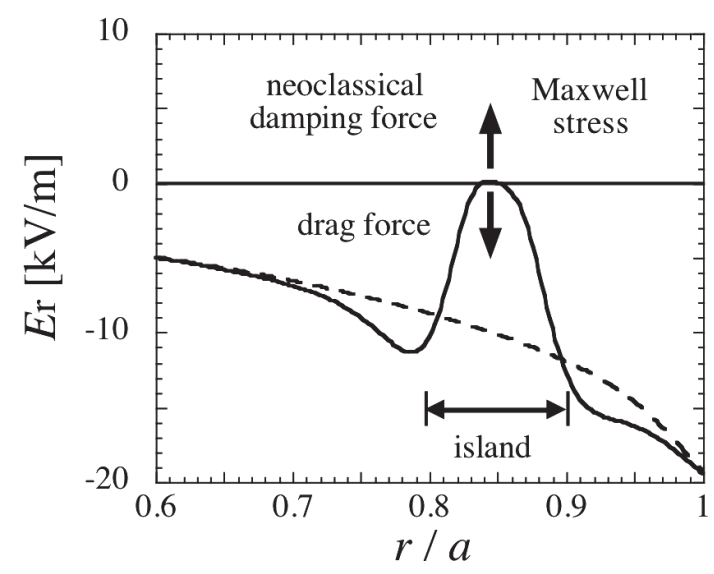

Fig. 2 Radial profiles of radial electric fields with $\mu_{\text {ano }}=5 \mathrm{~m}^{2} / \mathrm{s}$. Dashed and solid lines indicate the profile with $w_{\text {vac }}=$ $0 \mathrm{~cm}$ and that with $w_{\mathrm{vac}}=7.4 \mathrm{~cm}$, respectively.

layer width. If the neoclassical particle fluxes are excluded in Eq. (3), there does not exist the bifurcation in the saturation state, $w=w_{\text {vac }}$. When non-rotating magnetic islands are excited, the radial electric field inside magnetic islands is simultaneously damped (Fig. 2). Although the unperturbed radial electric fields are mostly determined by the ambipolar condition, $\Gamma_{\mathrm{i}}^{\text {neo }}=\Gamma_{\mathrm{e}}^{\text {neo }}$, the balance among three terms on the RHS of Eq. (3) becomes essential in the presence of magnetic islands. Since density and temperature gradients are flattened inside magnetic islands, there is no driving source of the neoclassical particle diffusion. In consequence, the coupling of Maxwell stress and neoclassical particle fluxes tends to damp the radial electric field inside magnetic islands, which is similar to the damping pendulum motion. While, the drag force acts in the opposite direction, and tends to sustain the radial electric field.

In summary, the forced magnetic reconnection in helical plasmas is investigated. It is found that the saturation 
state shows the bifurcation, when the poloidal rotation due to the neoclassical particle diffusion is taken into account. Detailed analyses and the comparison with experimental observations will be examined in the future work.

One of the authors, S. Nishimura, thanks Professor H. Sugama for fruitful discussions. This work is partially supported by a Grant-in-Aid for Scientific Research (S) (21224014) and (B) $(19360415,19360418)$ and by the collaboration program of the National Institute for Fusion Science, Japan (NIFS10KTAT048, NIFS09KDAD009). We also acknowledge the collaboration program of the Research Institute for Applied Mechanics of Kyushu University.

[1] Y. Narushima et al., Nucl. Fusion 48, 075010 (2008).
[2] K. Ida et al., Phys. Rev. Lett. 100, 045003 (2008).

[3] T. Estrada et al., Nucl. Fusion 47, 305 (2007).

[4] Y. Suzuki et al., Nucl. Fusion 46, L19 (2006).

[5] R. Fitzpatrick, Phys. Plasmas 5, 3325 (1998).

[6] S. Toda et al., Nucl. Fusion 47, 914 (2007).

[7] R.D. Hazeltine and J.D. Meiss, Plasma Confinement, Frontiers in Physics (Addison-Wesley, Redwood City, CA, 1992).

[8] K.C. Shaing, Phys. Fluids 27, 1567 (1984).

[9] P.H. Rutherford, Phys. Fluids 16, 1903 (1973).

[10] H.S. Hahm and R.M. Kulsrud, Phys. Fluids 28, 2412 (1985).

[11] A.I. Smolyakov, Plasma Phys. Control. Fusion 35, 657 (1993).

[12] K. Ida et al., Nucl. Fusion 44, 290 (2004).

[13] S. Nishimura et al., Nucl. Fusion 50, 054007 (2010). 